\title{
Methode zur jodometrischen Bestimmung von Silber, basiert auf der Reduktionswirkung von Kaliumarsenit.
}

\author{
Von \\ Rowland S. Bosworth. ${ }^{1}$
}

Es ist bekannt, dafs eine ammoniakalische Silberarsenitlösung metallisches Silber abscheidet, wenn man das Ammoniak durch Kochen entfernt. Bei dieser Reaktion wird gleichzeitig mit der Reduktion des Silbersalzes die arsenige Säure zur Arsensäure oxydiert nach der Gleichung:

$$
2 \mathrm{Ag}_{2} \mathrm{O}+\mathrm{As}_{2} \mathrm{O}_{3}=\mathrm{As}_{2} \mathrm{O}_{5}+4 \mathrm{Ag} \text {. }
$$

In der vorliegenden Mitteilung sind einige Untersuchungen beschrieben, die zum $Z_{\text {wecke ausgeführt wurden, festzustellen, ob diese }}$ Reaktion quantitativ verläuft, und ob man darauf eine schnelle jodometrische Methode zur Bestimmung des Silbers gründen könnte.

Die Versuche wurden zuerst mit einer Silbernitratlösung von bekanntem Gehalt nach folgendem Plan ausgeführt. Zu einer bestimmten Menge der Silbernitratlösung wurde ein gegebenes Volumen einer Kaliumarsenitlösung von bekanntem Gehalt hinzugesetzt, und zwar mehr als erforderlich war, um das rorhandene Silbersalz zu reduzieren. Hierauf fügte man hinreichend viel Ammoniak $\mathrm{zu}, \mathrm{um}$ den Niederschlag aufzulösen, verdünnte die Lösung auf $100 \mathrm{ccm}$ und kochte sie so lange, bis in den entweichenden Dämpfen mit Lackmus keine Spur Ammoniak mehr zu erkennen war. Die Lösung, aus der sich metallisches Silber abgeschieden hatte, wurde filtriert, abgekühlt und schwach sauer gemacht, um jede etwa verbleibende Spur Ammoniak zu neutralisieren. Nach Zusatz von Natriumbicarbonat titrierte man das überschüssige Kaliumarsenit mit $1 / 10^{-n o r m . ~}$ Jodlösung. Der Silberwert des verbrauchten Jods wurde von dem des ursprünglich angewandten Kaliumarsenits abgezogen und die Differenz als Mafs für das vorhandene Silber betrachtet. In Tabelle 1 sind unter A die Ergebnisse der in der beschriebenen Weise angestellten Versuche zusammengestellt.

1 Aus dem Amer. Journ. Sci. (Sill.) ins Deutsche übertragen von I. KopperBerlin. 
Tabelle 1.

\begin{tabular}{|c|c|c|c|c|c|c|}
\hline $\begin{array}{c}\text { Angew. } \\
\text { Silber } \\
\mathrm{g}\end{array}$ & $\begin{array}{l}\text { Zuges } \\
\text { ccm }\end{array}$ & $\begin{array}{l}\text { t. } \mathrm{KH}_{2} \mathrm{AsO}_{3} \\
\text { Silberwert } \\
\mathrm{g}\end{array}$ & $\begin{array}{l}\text { Ver } \\
\text { cem }\end{array}$ & $\begin{array}{c}\text { uchtes } J_{2} \\
\text { Silberwert } \\
g\end{array}$ & $\begin{array}{l}\text { Gef. } \\
\text { Silber } \\
\mathrm{g}\end{array}$ & $\begin{array}{c}\text { Fehler; } \\
\text { bezogen auf } \\
\text { Silber }\end{array}$ \\
\hline \multicolumn{7}{|c|}{$\mathrm{A}, \mathrm{B}$} \\
\hline 0.1054 & 20 & 0.2000 & 8.53 & 0.0949 & 0.1051 & -0.0003 \\
\hline 0.1054 & 20 & 0.2000 & 8.52 & 0.0948 & 0.1052 & -0.0002 \\
\hline 0.1054 & 30 & 0.3000 & 17.42 & 0.1939 & 0.1061 & +0.0007 \\
\hline 0.1159 & 20 & 0.2000 & 7.60 & 0.0846 & 0.1154 & -0.0005 \\
\hline 0.1054 & 21 & 0.2100 & 9.37 & 0.1043 & 0.1057 & +0.0003 \\
\hline 0.1054 & 20 & 0.2000 & 8.48 & 0.0944 & 0.1056 & +0.0002 \\
\hline \multicolumn{7}{|c|}{$\mathrm{NaHCO}_{3}$, Filtration. } \\
\hline 0.1054 & 15 & 0.1618 & 5.65 & 0.0571 & 0.1047 & -0.0007 \\
\hline 0.1054 & 23 & 0.2481 & 14.14 & 0.1430 & 0.1051 & -0.0003 \\
\hline 0.1054 & 12 & 0.1295 & 2.40 & 0.0243 & 0.1052 & -0.0002 \\
\hline 0.1054 & 15 & 0.1618 & 5.60 & 0.0566 & 0.1052 & -0.0002 \\
\hline 0.1054 & 15 & 0.1618 & 5.55 & 0.0561 & 0.1057 & +0.0003 \\
\hline 0.1054 & 20 & 0.2158 & 10.91 & 0.1104 & 0.1054 & \pm 0.0000 \\
\hline 0.2635 & 35 & 0.3770 & 11.83 & 0.1146 & 0.2630 & -0.0005 \\
\hline
\end{tabular}

Sodann wurde versucht, Natriumbicarbonat zur Erzeugung der Alkalität an Stelle von Ammoniak zu benutzen. Das folgende Verfahren kam zur Anwendung. $\mathrm{Zu}$ einer Lösung von Silbernitrat wurde ein Überschuis von Kaliumarsenitlösung von bekanntem Gehalt gegeben. Das Gemisch wurde alkalisch gemacht durch $25 \mathrm{ccm}$ einer gesättigten Lösung von Natriumbicarbonat, dann auf $100 \mathrm{ccm}$ verdünnt und gekocht, bis der Silberarsenitniederschlag sich in metallisches Silber verwandelt hatte. Die Lösung wurde dann filtriert und angesäuert, um die beim Kochen aus Bicarbonat gebildeten neutralen Carbonate zu zersetzen. Schlielslich wurde die Lösung mit Natriumbicarbonat alkalisch gemacht und das vorhandene Kaliumarsenit mit 1/10-norm. Jodlösung titriert. Die Einzelheiten dieser Versuche sind in Tabelle 1 unter B angeführt.

Da der Niederschlag von metallischem Silber bei den Versuchsreihen $A$ und $B$ sich gut zusammengeballt hatte, so führte man Versuche aus, bei denen die Filtration fortfiel und die Titration in Gegenwart des Niederschlages stattfand. Die in Tabelle 2 unter A und $B$ aufgeführten Ergebnisse zeigen, dals der Niederschlag keinen merklichen Finflufs auf die Titration ausübt. Wenn Salpetersäure in der Lösung vorhanden wäre, was gewöhnlich bei der Analyse der Fall ist, so würde sie durch Zusatz des Alkalis in Nitrat verwandelt 
werden. Um $z u$ beweisen, dafs die Gegenwart eiuer beträchtlichen Natriumnitratmenge die Reduktion des Silbersalzes nicht hindert, wurden Bestimmungen nach Zusatz von $2 \mathrm{~g}$ dieses Stoffes ausgeführt. Hierbei ergaben sich gleichmälsig gute Resultate, wie die Tabelle $2 \mathrm{C}$ zeigt.

Tabelle 2.

\begin{tabular}{c|c|c|c|c|c|c}
\hline \hline Angew. & \multicolumn{2}{|c|}{ Zugesetzt. $\mathrm{KH}_{2} \mathrm{AsO}_{3}$} & \multicolumn{2}{|c|}{ Verbrauchtes $\mathrm{J}_{2}$} & Gef. & Fehler; \\
Silber & & Silberwert & & Silberwert & Silber & bezogen auf \\
$\mathrm{g}$ & $\mathrm{ccm}$ & $\mathrm{g}$ & $\mathrm{ccm}$ & $\mathrm{g}$ & $\mathrm{g}$ & Silber \\
\hline \hline
\end{tabular}

$\mathrm{NH}_{4} \mathrm{OH}$. Titration erfolgte in Gegenwart des Niederschlages.

\begin{tabular}{l|l|l|r|r|r|r}
0.1054 & 20 & 02000 & 8.55 & 0.0952 & 0.1048 & -0.0006 \\
01054 & 20 & 0.2000 & 8.50 & 0.0916 & 0.1054 & \pm 0.0000 \\
0.1054 & 23 & 0.2300 & 11.28 & 0.1256 & 0.1044 & -0.0010 \\
0.1054 & 20 & 0.2000 & 8.45 & 00941 & 0.1059 & +0.0005 \\
0.1054 & 20 & 0.2000 & 8.48 & 0.0944 & 0.1056 & +0.0002
\end{tabular}

$B$

$\mathrm{NaHCO}_{3}$. Titration erfolgte in Gegenwart des Niederschlages.

\begin{tabular}{l|l|l|l|l|l|l}
0.1054 & 18 & 0.1800 & 6.80 & 0.0757 & 0.1043 & -0.0011 \\
0.1054 & 17 & 0.1700 & 5.81 & 0.0617 & 0.1053 & -0.0001 \\
0.105 & 15 & 0.1500 & 4.00 & 0.0445 & 0.1055 & +0.0001 \\
0.1054 & 21 & 0.2100 & 9.45 & 0.1052 & 0.1048 & -0.0006 \\
0.1054 & 25 & 0.2500 & 13.00 & 0.1447 & 0.1053 & -0.0001 \\
0.1054 & 31 & 0.3100 & 18.40 & 0.2048 & 0.1052 & -0.0002
\end{tabular}

$\mathrm{NaHCO}_{3}$. $2 \mathrm{~g} \mathrm{NaNO}_{3}$. Titration erfolgte in Gegenwart des Niederschlages.

\begin{tabular}{l|l|l|r|l|l|l}
0.0949 & 21 & 0.2100 & 10.42 & 0.1160 & 0.0940 & -0.0009 \\
0.1054 & 21 & 0.2100 & 9.43 & 0.1050 & 0.1050 & -0.0004 \\
0.1265 & 20 & 0.2000 & 660 & 0.0735 & 0.1265 & \pm 0.0000 \\
0.1686 & 21 & 0.2100 & 3.80 & 0.0432 & 0.1678 & -0.0008 \\
0.1054 & 15 & 0.1500 & 4.08 & 0.0454 & 0.1046 & -0.0008
\end{tabular}

$\mathrm{Da}$ es oft erforderlich ist, bei der Analyse Silber neben Kupfer und Blei zu bestimmen, und da Silber von diesen beiden Metallen durch Fällung mit Chlorwasserstoffsäure getrennt werden kann, schien es wünschenswert, auch die Reduktion des Silbers in Form von Chlorid auszuführen. Es wurden demnach die folgenden Versuche ausgeführt, deren Einzelheiten in Tabelle 3 unter A angegeben sind. Aus einer bekannten Menge Silberlösung wurde das Chlorsilber mit Salzsäure gefällt und über Asbest abgesaugt. Den Niederschlag löste man sodann vollständig in starkem Ammoniak auf, verdünnte die Lösung auf $100 \mathrm{ccm}$ und vollzog die Reduktion durch Zusatz eines Überschusses von Kaliumarsenitlösung mit bekanntem Gehalt and Kochen der entstehenden Lösung. Den Überschufs von Kalium- 
arsenit titrierte man sodann nach dem vorher beschriebenen Verfahren. In Tabelle 3 sind unter B die Einzelheiten ahnlicher Versuche angeführt, bei denen man das Silber von $0.09 \mathrm{~g}$ Kupfer trennte.

Tabelle 3.

\begin{tabular}{|c|c|c|c|c|c|c|}
\hline $\begin{array}{l}\text { Angew. } \\
\text { Silber } \\
\text { g }\end{array}$ & $\begin{array}{l}\text { Zuges } \\
\mathrm{ccm}\end{array}$ & $\begin{array}{l}\text { K. } \mathrm{KH}_{2} \mathrm{AsO}_{3} \\
\text { Silberwert }\end{array}$ & $\begin{array}{l}\text { Ver } \\
\text { cem }\end{array}$ & $\begin{array}{l}\text { uchtes } J_{2} \\
\text { Silberwert } \\
g\end{array}$ & $\begin{array}{l}\text { Gef. } \\
\text { Silber } \\
\mathrm{g}\end{array}$ & $\begin{array}{c}\text { Fehler; } \\
\text { bezogen auf } \\
\text { Silber }\end{array}$ \\
\hline
\end{tabular}

A

Reduktion von gefälltem Silberchlorid.

\begin{tabular}{l|l|l|l|l|l|l}
0.1017 & 15 & 0.1619 & 5.40 & 0.0599 & 0.1020 & +0.0003 \\
0.1017 & 15 & 0.1619 & 5.44 & 0.0603 & 0.1016 & -0.0001 \\
0.1017 & 15 & 0.1619 & 5.40 & 0.0599 & 0.1020 & +0.0003 \\
0.1017 & 15 & 0.1619 & 5.42 & 0.0601 & 0.1018 & +0.0001 \\
01017 & 17 & 0.1834 & 7.44 & 0.0825 & 0.1009 & -0.0008
\end{tabular}

$B$

Reduktion von $\mathrm{AgCl}$, das neben $0.09 \mathrm{~g}$ Kupfer ausgefällt war.

\begin{tabular}{l|l|l|l|l|l|r}
0.1017 & 15 & 0.1619 & 5.41 & 0.0600 & 0.1019 & +0.0002 \\
0.1017 & 15 & 0.1619 & 5.44 & 0.0603 & 0.1016 & -0.0001 \\
0.1017 & 15 & 0.1619 & 5.39 & 0.0598 & 0.1021 & +0.0004
\end{tabular}

Reduktion von $\mathrm{AgCl}$, das neben $0.2 \mathrm{~g}$ Blei gefält war.

$$
\begin{array}{l|l|l|l|l|l|l}
0.1220 & 16 & 0.1726 & 4.57 & 0.507 & 0.1219 & -0.0001 \\
0.1108 & 15 & 0.1619 & 4.60 & 0.0510 & 0.1109 & +0.0001
\end{array}
$$

Reduktion von $\mathrm{AgCl}$, das aus einer Lösung mit $0.09 \mathrm{~g}$ Kupfer und $0.2 \mathrm{~g}$ Blei

$$
\begin{array}{l|lll|l|l|l|l|l}
0.1017 & 15 & \mid & 0.1619 & 5.45 & 0.0604 & 0.1015 & -0.0002
\end{array}
$$

Die Versuche in Tabelle 3 unter $\mathrm{C}$ zeigen die Trennung des Silbers von $0.2 \mathrm{~g}$ Blei und die Versuche unter $\mathrm{D}$ illustrieren die Trennung des Silbers von $0.09 \mathrm{~g}$ Kupfer und $0.2 \mathrm{~g}$ Blei.

Aus den hier mitgeteilten Resultaten ergibt sich, dafs das Silber sowohl in Lösung wie in Form von gefälltem Chlorid bestimmt werden kann durch Zusatz eines Überschusses von Kaliumarsenitlösung mit bekanntem Gehalt, Kochen der alkalischen Lösung zur Vollendung der Reduktion des metallischen Silbers und Titration des überschüssigen Kaliumarsenits mit Jod. Der Silberwert des verbrauchten Jods wird von dem des ursprünglich angewendeten Kaliumarsenits abgezogen und die Differenz gibt dann die Menge des vorhandenen Silbers an.

New Haven, U.S.A., The Kent Chemical Laboratory of Yale University.

Bei der Redaktion eingegangen am 11. Juli 1909 